\title{
RECEPCIÓN DE MAQUIAVELO EN ESPAÑA EN LOS SIGLOS XVI Y XVII
}

\author{
Alfonso Tomás Sobrino González \\ UNED \\ alfonsosobrinogonzalez@gmail.com
}

\section{RESUMEN}

El presente trabajo discute y argumenta sobre la posibilidad y la forma en que la obra de Maquiavelo repercute en el panorama literario político español en los siglos XVI y xviı. Se centra, primordialmente, en una visión crítica de dos argumentos sostenidos para negar la presencia del pensamiento maquiaveliano en dicho lugar y momento: el escaso número de traducciones al castellano halladas y la intensa actividad antimaquiaveliana de la Inquisición española aun cuando ello no impidió el tratamiento de Maquiavelo por parte de figuras literarias españolas de primer orden e impacto político, tales como Juan de Mariana, Quevedo, Gracián, Saavedra Fajardo, Ribadeneyra, Mártir Rizo, Juan Ginés de Sepúlveda y otros.

Palabras clave: Maquiavelo, maquiavelismo, antimaquiavelismo, España, siglos xvi y xviI.

\section{MACHIAVELLI'S PERCEPTION IN SPAIN \\ DURING THE $16^{\mathrm{TH}}$ AND $17^{\mathrm{TH}}$ CENTURIES}

\section{Abstract}

This paper discusses and argues the possibility and the way in which Machiavelli's work affects the Spanish political and literary perspective during the 16th and 17th centuries. It focuses primarily on a critical view of two arguments held to deny the presence of the Machiavellian line of thought in that particular place and time: the scarce number of translations into Spanish found and the intense anti- Machiavellian activity of the Spanish Inquisition even though it did not hinder Machiavelli's treatment by leading literary figures and political impact, such as Juan de Mariana, Quevedo, Gracián, Saavedra Fajardo, Ribadeneyra, Mártir Rizo, Juan Ginés de Sepúlveda, and others.

KeYwORDs: Machiavelli, machiavelism, antimachiavelism, Spain, 16th and 17th century.

DOI: https://doi.org/10.25145/j.laguna.2019.45.02

Revista Laguna, 45; diciembre 2019, pp. 35-46; ISSN: e-2530-8351 


\section{INTRODUCCIÓN}

No resulta difícil encontrarse ante la idea que niega, no solo el impacto, sino la presencia de las obras de Maquiavelo en España durante los siglos XVI y XVII. Fundamentalmente, esta postura se asienta en dos principios, considerados rotundos e indiscutibles para quienes defienden dicha idea: en primer lugar, el poder de la Inquisición española durante este periodo, cuyos planteamientos confrontaban radicalmente con el pensamiento del secretario; en segundo lugar, como efecto del anterior, la escasez de traducciones españolas de las obras de Maquiavelo.

Por un lado, aquí nos preguntamos cómo es posible que la obra de Maquiavelo no haya influido en la España de los siglos XVI y xvıI, y a la vez sintamos que atraviesa todo eje temático en torno a la reflexión ético-política erigiéndose, por tanto, como punto de referencia obligado y fundamental ${ }^{1}$. Bien es cierto que en la literatura política española se hallan antecedentes ${ }^{2}$ a Maquiavelo; sin embargo, estos no alcanzan la sistematicidad del pensamiento político que aparece en la cultura española durante los siglos inmediatamente posteriores a la obra del florentino. Con todo, se niega el influjo de Maquiavelo argumentando que no existen suficientes traducciones de su obra al castellano como para haber impactado directamente en la España de los siglos XVI y XVII. Por otro lado, aun cuando la posición de la literatura política española durante los siglos XVI y XVII parece netamente antimaquiaveliana ${ }^{3}$, lo cierto es que rezuma maquiavelismo por todos sus poros. Si a ello le añadimos que J.A. Maravall ${ }^{4}$ y H. Puigdomènech ${ }^{5}$, entre otros, consideran del todo injustificada la posición que niega el impacto y la influencia de Maquiavelo en España durante

${ }^{1}$ Por ejemplo, en De rege et regis institutione (1559) del padre jesuita Juan de Mariana; en Política de Dios y gobierno de Cristo (1617) de Quevedo; en El héroe (1637), El político (1640), Oráculo manual y arte de la prudencia (1647), y El criticón (1651) de Gracián; en las Empresas politicas (1640) de Saavedra Fajardo; en el Tratado de la Religión y virtudes que debe tener un Principe christiano para gobernar y conservar sus Estados contra lo que Nicolás Maquiavelo y los politicos de este tiempo enseñan (1595) de Ribadeneyra; Mártir Rizo y su Norte de Príncipes (1626); Juan Ginés de Sepúlveda y su Democrates Primus, (1550); Baltasar Álamos de Barrientos en su Discurso político al rey Felipe II al comienzo de su reinado (1589); Juan Alfonso de Lancina y sus Comentarios politicos a los Annales de Cayo Vero Cornelio Tácito (1687); o, incluso, Juan Luis Vives, en diversas obras.

2 Por poner solamente algunos ejemplos, en 1441, Diego de Valera escribe al rey Juan II de Castilla «Mucha prudencia es a tiempo desimular las cosas». Sobre 1454, Rodrigo Sánchez de Arévalo sostiene que en la guerra son lícitos, una vez comenzada, los fraudes y ardides. García de Palacio sostendrá el mismo principio y Ginés de Sepúlveda afirma que, por derecho divino, natural y de gentes, en la guerra están permitidos asechanzas, simulaciones, engaños. A ellos unimos al arzobispo don Sancho de Rojas, cuando afirma que «a fin de mandar, regir e aun de se vengar, algunas veces usava de algunas cabtelas e artes", tal como cita Maravall, J.A., en Estudios de Historia del Pensamiento Español-Serie tercera-El siglo del Barroco, p. 42.

3 Sobre todo, la posición jesuita.

${ }^{4}$ J.A. Maravall, Teoria española del Estado en el siglo XVII, Madrid, Instituto de Estudios Constitucionales, 1997.

5 Puigdomènech, H., Maquiavelo en España. Presencia de sus obras en los siglos XVI y XVII, Madrid, Fundación Universitaria Española, 1988. 
los siglos XVI y XVII, no queda más remedio que examinar rigurosamente la validez de los criterios y los argumentos utilizados.

Por lo que respecta a las escasas traducciones encontradas cabe plantearse cuántos ejemplares fueron expurgados, y de ello pueden encontrarse algunos rastros bibliotecarios. Con relación al poder innegable que adquirió la Inquisición española, parece necesario circunscribirla no solo a su ámbito de actuación práctica, sino también a sus fundamentos teóricos, es decir, tanto en su dimensión interna dentro del territorio español, como en su dimensión filial, dependiente de la Inquisición romana. En este último aspecto habremos de estudiar la posición de la Iglesia, y del papado, ante Maquiavelo, su obra y su pensamiento.

\section{TRADUCCIONES DE MAQUIAVELO}

Ya hemos anotado que en el abordaje de esta cuestión opera habitualmente un reduccionismo lógico consistente en negar la influencia de Maquiavelo en España, debido a la prohibición que sobre Maquiavelo había establecido la Inquisición y, en consecuencia, el escaso número de traducciones al castellano habidas. Si interpretamos el impacto literario de nuestro autor basándonos en el número de traducciones el resultado sería, ciertamente, un auténtico descalabro, pues apenas se cuentan ejemplares traducidos. Sin embargo, hay que investigar los motivos.

Siguiendo a Puigdomènech ${ }^{6}$, sabemos que 1521 es el año en que se imprime en Italia por primera vez la obra de Maquiavelo. Sin embargo, la primera impresión de El principe en castellano no verá la luz hasta 1854 , gracias a la imprenta de don José Trujillo hijo. Entre ambas no hay vestigios de existencia más que de dos ediciones de una traducción de los Discorsi y otra de El arte de la guerra, que además no se presenta como traducción. No obstante, y como se verá más adelante, este hecho no es atribuible a la supuesta prohibición inquisitorial sobre la obra de nuestro autor. Empero, debe haber una explicación. Y es que, como señala Bertini «In realtà le versioni degli scritti del "secretario fiorentino" non dovettero essere molto numerose, grazie allá familiarità che gli spagnoli avevano coll'italiano»?.

Lo cierto es que el pensamiento maquiaveliano había permeado en las concepciones políticas, con mayor o menor gravedad, de las grandes potencias europeas, véase al menos Francia, Inglaterra (donde abundan las traducciones) y España. También es cierto que, durante los siglos XV y XVI, existía un estrecho vínculo entre España e Italia y, de hecho, parte importante de la actual Italia pertenecía al Imperio español. No en vano, para los servidores de la Corona o la Iglesia españolas, era inexcusable en su formación el paso por Italia, por no hablar de los interesados en las

\footnotetext{
${ }^{6}$ Cfr. op. cit.

7 Bertini, G.M. (1946), «La fortuna de Machiavelli in Spagna», Quaderni ibero-americani, II, p. 21.
} 
ciencias o el arte. Quizás por ello escribe el duque de Sessa ${ }^{8}$ una carta al Consejo de la Inquisición, el 9 de noviembre de 1584 primero, y el 1 de junio de 1585 después, solicitando permiso para publicar algunas traducciones de Maquiavelo, estimándolas de provecho. No puede obviarse que uno de los paradigmas ideales de hombre renacentista se halla caracterizado por ser hombre de letras y de armas, cultivador tanto de las virtudes bélicas como las humanísticas. En otras palabras, aquellos a quienes habría de interesar la obra maquiaveliana, ineludiblemente se habrían formado en Italia. Prueba de ello es el elevado número de estudiantes y profesores españoles que coexistían en las universidades italianas, incluso más allá de 1559, tras la llamada al regreso a España de cuantos estudiaban o enseñaban más allá de las fronteras españolas. De hecho, se estima que cerca del veinte por ciento de la población romana de principios del siglo XVI era española. Españoles formados o en proceso de formación, intelectuales interesados en la política y/o en las artes militares, aspectos ambos entrelazados en la compleja obra de Maquiavelo. Cabe, por ello, suponer que en el equipaje de regreso de estos hombres a Espańa viajaba Maquiavelo, ya en papel, ya integrado en el pensamiento, sin necesidad alguna de traducción. En esta dirección, cobra especial relevancia el papel de los intelectuales a cargo de las embajadas españolas en las distintas repúblicas italianas. Posiblemente la más interesante sea la de Venecia, uno de los mayores centros editoriales europeos del momento. En ella ubicamos al bibliófilo Diego Cristóbal de Salazar, secretario del embajador, cuando Maquiavelo no está aún prohibido por el Índice inquisitorial español. Salazar compró y envió libros por encargo a España, incluso al mismo Felipe II y a sus allegados, pero tan importante para la difusión de la literatura italiana como Salazar, o quizás más trascendente todavía, fue Alfonso de Ulloa, quien editó y tradujo numerosas obras del italiano al español, aun cuando de entre las obras que editó no encontramos ninguna de Maquiavelo.

Sin embargo, esta no parece ser la única vía de entrada de la obra del secretario. A finales del siglo xv y principios del Xvi existe un buen número de italianos circulando, o incluso ya establecidos, en territorio español. La mayoría son mercaderes, y entre ellos los hay especializados en libros, negocio al alza desde la invención de la imprenta y la incipiente difusión editorial. La actividad de los libreros no se reduce exclusivamente a comprar o vender ejemplares, sino que la rebasa, introduciéndose en una dimensión intelectual. De igual modo sucede con los editores, cuya función no consiste únicamente en editar e imprimir, sino que con la elección de aquello que editan sirven de vía de difusión del pensamiento. A este respecto cabe destacar el establecimiento en Medina del Campo de Guillermo Millis, quien a la postre será el editor de Maquiavelo en España. Sin embargo, aunque la mayoría de los italianos afincados o en tránsito por España son mercaderes, hay un reducido número de intelectuales cuya influencia en los círculos palaciegos es profunda. Profesores de universidad, cortesanos reales, preceptores de princesas, artistas cola-

\footnotetext{
${ }^{8}$ De ello se hacen eco tanto Puigdomènech, H. como Bertini, G.M. en sus correspondientes estudios sobre la cuestión.
} 
boradores en la construcción del monasterio de San Lorenzo de El Escorial. Todos ellos, sin duda, sirven de vía de difusión de la cultura y la lengua italianas. En este sentido, cobra especial relevancia saber que hubo numerosas impresiones y ediciones de, al menos, dos diccionarios italiano-español. Este hecho no parece casual si consideramos que el conocimiento del francés y del toscano (para declamar a Petrarca) eran toques distintivos de buenos modos para un caballero, a pesar de la opinión cervantina, que estima innecesario esforzarse en aprender un idioma tan fácil.

En definitiva, sostenemos aquí que la escasez de obras maquiavelianas traducidas al español durante el siglo xvi no resulta un impedimento para el conocimiento, el impacto y la propagación de su pensamiento por territorio espańol. Por todo lo expuesto anteriormente, manifestamos que las traducciones eran innecesarias para el público potencial de nuestro autor. En otras palabras, la escasez de traducciones de Maquiavelo no implica necesariamente el desconocimiento de su pensamiento y de su obra. Las traducciones no son indefectibles para el conocimiento. Sin embargo, la presencia de su obra sí lo es.

En referencia a las obras que sí encontramos traducidas debemos mencionar varias traducciones manuscritas, datadas de finales del siglo XVI y principios de siglo XVII, y varias ediciones de dos de sus obras: El arte de la guerra, parafraseado literalmente por el capitán Diego de Salazar en 1535 o $1536^{\circ}$ bajo el título De re militari, y dos ediciones de los Discorsi hechas por Ottevante y editadas, como mencionamos anteriormente, por Guillermo Millis en Medina del Campo en 1552 y 1555. El impacto de las obras editadas debió ser de gran calado, la primera de ellas se encuentra con cierta facilidad en bibliotecas de la época y la segunda se editó en dos ocasiones en un corto espacio de tiempo. Ya en 1680 encontramos ejemplares de toda la obra maquiaveliana en la Biblioteca Nacional de Madrid ${ }^{10}$, pero ¿sería posible afirmar su presencia escrita en lengua toscana, lengua dominada, como acabamos de mostrar, por aquellos a quienes interesaría la lectura del florentino, antes de esta fecha?

Guiados por la incipiente labor investigadora de Bertini ${ }^{11}$, así como su posterior desarrollo, extraordinario y exhaustivo de Maravall ${ }^{12}$ y Puigdomènech ${ }^{13}$, tenemos referencias de ciertos registros bibliotecarios de los años precedentes. Su labor se centra, no con pocas dificultades, en el estudio de documentos históricos, véanse: en la Biblioteca Nacional de Madrid ${ }^{14}$, inventarios, documentos de subastas de bienes, catálogos de bibliotecas e inventarios de obras prohibidas expurgadas por la Inqui-

9 Maravall, J.A., en Estudios de Historia del Pensamiento Español, se inclina por 1535, mientas que Puigdomènech (supra) lo hace por 1536.

10 Maravall, J.A., op. cit., p. 48

11 Cfr. Bertini, G.M. (1946), "La fortuna de Machiavelli in Spagna», Quaderni ibero-americani, II, pp. 21-22, 25-26.

12 Cfr. Maravall, José Antonio, «Maquiavelo y el maquiavelismo en España», Boletín de la Real Academia de la Historia, (Madrid), CLXv (1969) 183-218.

13 Supra.

${ }^{14}$ Según Bertini, op. cit. p. 22, «numerose [obras] dovettero invece essere le edizioni italiane diffuse in Spagna, se la sola Biblioteca Nazionale di Madrid ne possiede ben sei del sec. Xvi e più ancora dei secoli successivi». 
sición española ubicados en el Archivo Histórico Nacional de los siglos XVI y XVII, dejando de lado las citas, directas o indirectas. A partir de estos datos se traza un análisis tanto de la personalidad de los propietarios de las obras de Maquiavelo como de la posible circulación de las obras del florentino en los círculos frecuentados por dichos propietarios. Todos estos personajes han sido incluidos en una de estas cuatro categorías: personajes regios, eclesiásticos, nobles o intelectuales.

Para estudiar a los primeros se toman como referencia las bibliotecas del duque de Calabria en 1550, la de Carlos V y la de Felipe II, y en todas ellas se halla presencia maquiaveliana. Con respecto a las bibliotecas eclesiásticas se mencionan, por un lado, las de los cardenales don Francisco de Mendoza Bobadilla (1556), don Antonio de Aragón (1651), y don Pascual de Aragón (1677), los tres desempeñaron papeles diplomáticos y de gobierno, y el último fue, además, inquisidor general; por otro lado, la del arzobispo y virrey de Valencia, san Juan de Ribera (1611); y en último lugar las del inquisidor general don Diego de Arce (1666). De nuevo aparece la obra maquiaveliana en todas ellas. En lo tocante a las bibliotecas de nobles la lista de bibliotecas consultadas es más extensa. Se mencionan la biblioteca del tercer duque de Béjar (1544), la de don Diego Hurtado de Mendoza (1575), la del duque del Infantado en Guadalajara (1575), la del duque de Medinacelli (1575), la del conde-duque de Olivares y de San Lúcar (1625), la del conde de Benavente (1633), la de don Diego Sarmiento de Acuña, conde de Gondomar (1623-1634) y la del virrey don Pedro Antonio de Aragón. En todas ellas aparecen, de uno u otro modo, referencias a la posesión de obras de Maquiavelo, a excepción de la biblioteca del conde-duque de Olivares y de San Lúcar y la del conde de Benavente. A pesar de no encontrar estos rastros, por otros motivos es muy plausible que conocieran y/o poseyeran la obra de nuestro autor. Además, en las bibliotecas de intelectuales y artistas llama la atención que no se encuentra rastro documental patente de la obra maquiaveliana; no obstante, se encuentra la categoría "libros italianos» sin especificar, o títulos que podrían ser obras maquiavelianas en la biblioteca de Gonzalo Pérez, secretario de Felipe II (1574); la de don Luis Barahona de Soto (1595); la del Inca Garcilaso (1616); la de don Agustín de Villanueva (1621); la del doctor Gonzalo de Correas (1631); la de Lorenzo Ramírez del Prado (1660); la de Velázquez (1660); y la de Gaspar Murillo. Únicamente hay rastro documental en la biblioteca de don Vicencio Juan de Lastanosa (1684). Sin embargo, a todos ellos se les atribuye suficiente conocimiento de política, y específicamente clásica e italiana, como para ser conocedores de la obra de Maquiavelo. A la ya aludida difusión del plagio de Salazar se unen otros. De ello se deduce que no fue tanto la obra de Maquiavelo la que estuvo prohibida como su nombre. Además, la prohibición de la obra del florentino se hizo en España efectiva 24 años después de la romana, por lo que podría haberse extendido su obra con cierto desconocimiento por parte de las autoridades. Por último, cabe decir que, ya sea por la relevancia de los hombres propietarios de estas bibliotecas, ya por la ineficacia de los organismos administrativos españoles, la prohibición inquisitorial no impidió la circulación de estas obras. 


\section{LA POSTURA DE LA IGLESIA ANTE MAQUIAVELO, SU PENSAMIENTO Y SU OBRA}

Afirmar, de modo tajante y absoluto, que la posición de la Iglesia respecto de la obra y pensamiento de Maquiavelo es unívocamente contraria nos parece reduccionista. Aun cuando en términos generales es correcto pensar que la Iglesia adoptó una actitud antitética a los argumentos maquiavelianos y su actitud fue de claro enfrentamiento, hemos de conceder espacio para aclarar ciertos matices.

Inicialmente, anotamos la posición de Lefort ${ }^{15}$, alineado con Villari ${ }^{16} \mathrm{y}$ con Panella ${ }^{17}$, al advertir que «el papa autoriza la impresión de El Príncipe y los Discorsi mediante una cédula; un cardenal les concede su protección, otro acepta la dedicatoria del autor $\aleph^{18}$. A continuación, afirmamos que la actitud de los papas y de la Iglesia con respecto a la obra de Maquiavelo pasó por diversas etapas, reductibles a dos posiciones antagónicas. En un primer momento la Iglesia mantuvo una postura favorable; sin embargo, a partir de 1532 se mostrará abiertamente desfavorable, inclinándose paulatinamente hacia el rechazo y la condena absoluta de la obra del florentino por parte de la Inquisición romana.

Antes de entrar a analizar dichas etapas conviene recordar que el papado estuvo ocupado por miembros de la familia Médici, al menos entre los años 1513 y 1535 (a excepción del periodo que va del 9 de enero de 1522 al 14 de septiembre de 1523, ocupado por Adriano VI), por lo que las relaciones entre la Iglesia se confundían con las relaciones clientelares mediceas. En este sentido, como es habitual recordar, la indignante recompensa que recibió ${ }^{19}$ Maquiavelo al entregar El príncipe a los Médici parece mostrar desdén absoluto, cuando no rechazo, hacia nuestro autor. Sin embargo, estas relaciones eran más paradójicas de lo que cabe suponer.

Sea como fuere, en el primer periodo, fechado entre el destierro de Florencia en San Casciano y su muerte 1512-1527, la Iglesia mantuvo una actitud favorable hacia Maquiavelo. Tanto es así que, incluso, el papa León X (Giovanni di Lorenzo di Medici) mandó construir un teatro ex professo para la representación teatral de La mandrágora en 1520. Ello a pesar de que, como muestra Ridolfi ${ }^{20}$, Maquiavelo no era un personaje precisamente bien avenido con los Médici, por dos motivos fundamentalmente: primero, por haber sido miembro de la República florentina, adversa a los intereses familiares de estos; segundo, por haber sido sospechoso de participar en la conjura antimedicea de Boscoli, hecho por el cual estuvo encarcelado hasta la mediación del recién elegido papa León X. El mismo año de 1520 es contratado por el Estudio florentino, cuyo presidente es el cardenal Giulio di Giuliano di Médici (primo de León X), quien le encarga la redacción de las Istorie fio-

${ }^{15}$ Cfr. Lefort, C., Maquiavelo, lecturas de lo político, Madrid, Trotta, 2010.

16 Villari, P., Maquiavelo, su vida y su tiempo, México D.F., Biografías Gandesa, 1953.

17 Panella, A., Gli antimachiavellichi, Firenze, Sansoni, 1943.

${ }^{18}$ Lefort, C., op. cit., p. 19.

19 Apenas un par de botellas de vino.

${ }^{20}$ Cfr., Ridolfi, R., Vita de Niccolò Machiavelli, Sansoni Editore, Firenze, 1969. 
rentine. Esta obra, concluida y entregada en 1525 , fue dedicada al mismo Giulio di Giuliano di Médici, ya como papa Clemente VII, y por ella Maquiavelo fue recompensado con una donación de dinero y un aumento de salario. Además, es sabido que, cuando el propio Clemente VII decide enviar una legación, con su sobrino el cardenal Salviati, a la corte de Madrid, se piensa en Maquiavelo como secretario, aunque finalmente esta posibilidad no llegara a buen término. Una última prueba de la buena consideración que se tenía por Maquiavelo en el círculo mediceo-papal es su nombramiento como superintendente y canciller de los cinque Procuratori delle mura, institución creada a instancias del propio Maquiavelo por el papa.

Un año después muere Maquiavelo, pero Clemente VII sigue protegiendo su legado. Tanto es así que el impresor de Cámara pontificio Antonio Blado d'Asola (quien más tarde escribirá el Índice en el que se prohíbe por primera vez al florentino) obtiene el privilegio exclusivo del papa, con el apoyo de monseñor Gaddi y el cardenal Ridolfi, para reproducir la obra de Maquiavelo durante diez años. El mismo año de 1531, estando ya casi agotada esta edición, se concede un nuevo privilegio al florentino Giunta para la edición maquiaveliana, que dedicará a Ottaviano de Médici. Habrá que esperar quince años tras la publicación de El príncipe para vislumbrar la interpretación que se comienza a hacer de la obra del florentino, bajo el efecto de los avances de la herejía. Será entonces cuando el cardenal inglés «Reginald Pole [...] se indigna (y) apenas ha abordado la obra, ya la reconoce como escrita por la mano de Satán. Si Satán reinase sobre la tierra, no legaría otros preceptos a su hijo antes de cederle su reino» ${ }^{21}$. Posteriormente, el dominico italiano Ambrogio Caterino denunciará el ateísmo de los Discorsi y, a continuación, el obispo portugués Girolamo Osorio calificará el segundo capítulo del Libro in de los Discorsi como una «escandalosa apología del paganismo» ${ }^{22}$.

La ética cristiana, cuya inspiración en la vida social era omnipresente, venía a ser socavada por medio de una crítica al poder en términos secularizados y ello suprimía la referencia al plan divino del Creador en tanto en cuanto guía universal de la humanidad. Por ello, los ideólogos antimaquiavelistas se apresuran a embestir a su adversario con todo género de acusaciones, desde el ateísmo hasta la herejía, pasando por el satanismo. La desacralización de las relaciones sociales y la mundanización de la política no solo niegan el cristianismo, sino que son tomadas como una inaceptable subversión del orden social y moral.

${ }^{21}$ Lefort, C., op. cit., p. 19.

22 Idem. 


\section{LA ACTITUD DE LA INQUISICIÓN ESPAÑOLA RESPECTO A MAQUIAVELO}

Desde su fundación por los Reyes Católicos en 1478 es evidente, e innecesario discutir aquí, el poder fáctico que acumula la Inquisición española bajo el control directo de la monarquía española, durante de los siglos XVI y XVII. La autoridad de la monarquía, a este respecto, proporcionaba a la Inquisición una autonomía excepcional, hasta el punto de poder afirmar que existía una gran independencia frente a Roma. Los inquisidores generales españoles y, lógicamente, el Consejo de la Santa Inquisición, tenían como prioridad la observancia y obediencia a la monarquía en primer lugar y, en segundo lugar, a la Iglesia romana. Muchos son los hechos que lo prueban, y en lo que resulta pertinente a nuestro tema hallamos, de entre todos ellos, algunos de representatividad extraordinaria. Así lo refleja, por ejemplo, que mientras la Iglesia romana (a pesar de haber protegido inicialmente, como ya hemos visto, la obra de Maquiavelo por parte de monseñor Gatti y el cardenal Ridolfi) incluyó las obras del florentino en el Index librorum prohibitorum de Pablo IV de 1559, y «tras la condena definitiva en 1564 por el Concilio de Trento, se llegó a acusar a Maquiavelo de haber preparado la Reforma y ser ministro de Satán $»^{23}$, en España se publicó el Índice de Valdés en 1559 (ocho meses después del Índice de Pablo IV), en el que no existía referencia alguna a Maquiavelo, y habrá que esperar para su prohibición hasta 1583, cuando sea incluido en el Índice del cardenal Quiroga, donde se «rinnovava la precedente proibizione e citava esplicitamente la prima versione di un'opera di Machiavelli ${ }^{24}$. Todo ello, a pesar de las denuncias anteriores del cardenal inglés R. Pole en 1539 y el hispano-portugués Jerónimo de Ossorio en su obra De nobilitate christiana, fechada en 1542. Para mostrar la atención con la que se trataban los asuntos provenientes de Roma desde la Inquisición de Córdoba, Puigdomènech ${ }^{25}$ se hace eco de la carta enviada el 4 de abril de 1559 por el licenciado Villar, donde solicita a su superior orientación para saber cómo nos hemos de aver con tan larga prohibición. Ello revela tanto la independencia como la autonomía, con respecto a Roma, de la Inquisición española.

Ya hemos hecho referencia a la naturaleza inquisitorial, creada por la monarquía española bajo su control primordial directo. A ello hay que añadir que el inquisidor Valdés era un hombre del emperador, y que este había decidido proteger explícitamente para su traducción los Discorsi, desde 1550 y hasta diez años en adelante.

${ }^{23}$ Negro Pavón, Dalmacio, «Maquiavelo no era maquiavélico», en Schwartz Girón, Pedro (coord.), El Principe de Maquiavelo en el quinto centenario de su composición, p. 36, Madrid, España, Real Academia de las Ciencias Morales y Políticas.

${ }^{24}$ Se refiere aquí Bertini, G.M., op. cit., p. 21, a la traducción que realizó Guillermo de Millis en Medina del Campo en el año 1555 (ad. Opera di J. Lorenzo Otevanti) de Los discursos sobre la primera década de Tito Livio.

${ }^{25}$ En su artículo Maquiavelo y maquiavelismo en España. Siglos XVI y XVII, con el que se inicia el libro de Forte, J.M. y López Álvarez, P. (eds.), Maquiavelo y España. Maquiavelismo y antimaquiavelismo en España en los siglos XVI y XVII, Madrid, Biblioteca Nueva, 2008. p. 42. 
De hecho, el emperador Carlos V admitía leer con deleite dicha obra, que estaba, por otra parte, dedicada a su hijo, por entonces príncipe, Felipe. Como se ve, se ignora el Índice de Pablo IV publicado en 1559. Pero, además, tampoco se toma en cuenta el Índice Tridentino de 1564, donde Maquiavelo aparece condenado nuevamente. Será en 1584 cuando Maquiavelo será incluido en el Îndice de la Inquisición española, 25 años después de su prohibición en Roma. El Índice del inquisidor Quiroga contó con la influencia del padre Mariana, propulsor de la preponderancia de la Iglesia frente a cualquier Estado. Sin embargo, hubo intentos de secularizar el poder estatal y, con ello, solicitudes de suprimir algunas obras de Maquiavelo del Índice, por ejemplo, las del duque de Sessa, Antonio Folch de Cardona. Como es fácil suponer, cualquier intento de secularización, así como los instrumentos utilizados para ello, fueron combatidos fuertemente por la Inquisición española. En los partidarios de la política secularizada, se supone la aceptación maquiaveliana de independencia política y de autonomía del Estado frente a la Iglesia y la moral. Quizás, incluso, entre los seguidores de la política secularizada, la prohibición de Maquiavelo alentó más aún a su lectura.

En cualquier caso, tras el Índice de Quiroga de 1583, Maquiavelo será prohibido en todos los Índices, como así lo muestra el Índice de Sandoval y Rojas de 1612, el del inquisidor Zapata en 1632 y los posteriores. Sus obras serán buscadas y quemadas, en el peor de los casos, o requisadas en el mejor de ellos. Ello coadyuvó a su tráfico en el mercado negro, negocio ciertamente rentable entre los bibliotecarios y bibliófilos. Gracias a los registros y a las expropiaciones anotadas, expuestas anteriormente, podemos afirmar la presencia e influencia de las obras de Maquiavelo en España durante los siglos XVI y XVII.

Así las cosas, podemos afirmar que, aunque la Inquisición española expurgara e indizara las obras de Maquiavelo, lo hizo con lentitud y escaso éxito. Maquiavelo era bien conocido por políticos, estadistas, militares y nobles. Otra prueba de ello la hallamos en las deliberaciones del Consejo de Estado acerca de las consecuencias de la paz de Crépy, con la que España debía ceder el Milanesado o los Países Bajos. Lo habitual cuando se estudia este problema histórico es encontrar respuestas y explicaciones a la problemática religiosa del luteranismo. Sin embargo, a tenor de la información hallada en las actas de estas reuniones, y a pesar de los muchos religiosos que se hallaban en ellas, en el transcurso de estas deliberaciones no se hizo ninguna argumentación en términos religiosos, en alusión al problema luterano. Todas las intervenciones, independientemente de la opción que defendieran, se expresaron en términos relativos a razones teórico-políticas, radicalmente ligadas a la terminología maquiaveliana.

\section{CONCLUSIÓN}

En conclusión, sabemos que Maquiavelo no solo era bien conocido en España durante los siglos XVI y XVII, sino que la repercusión de su pensamiento reverbera en los numerosos tratadistas políticos y teólogos, y además el eco de sus obras se escucha entre los hombres de Estado, ya sean diplomáticos, ya gobernantes, ya reyes, sobre los que hemos indagado. 
Sabemos también que, a menudo, ha habido dudas acerca del impacto y el alcance del pensamiento maquiaveliano en España, básicamente por las ideas preconcebidas acerca de la España Moderna inquisitorial, cuya tendencia consiste en pensar que las obras de Maquiavelo no fueron leídas en España al estar incluidas en el Índice de libros prohibidos por la Iglesia católica. Esta interpretación, por lo demás, es absolutamente lógica si observamos el mantenimiento de la moral de orden cristiano en España durante los siglos XVI y XVII. Y, si a ello le unimos la escasez de traducciones castellanas de la obra de nuestro autor, la argumentación parece revestirse de una lógica infalible.

Sin embargo, el orden de lo lógico no siempre alcanza el orden de lo real, y mucho menos de la verdad. Por un lado, esperamos haber mostrado que de la escasez de traducciones al castellano de la obra maquiaveliana no se infiere necesariamente la ausencia de su huella e influencia en España durante los siglos trabajados. Por ello concluimos que la escasez de traducciones no resulta un argumento ni un criterio válido para negar la presencia, el impacto y el influjo de la obra maquiaveliana, pues pudieron ser otros los medios de propagación de su pensamiento. Por ejemplo, consideramos legítima la idea que defiende la difusión de su pensamiento desde las obras escritas en toscano, dado que no había necesidad de traducciones para el potencial lector de Maquiavelo, o para aquellos que se formaron en Italia, y además hemos acreditado la presencia de la obra de Maquiavelo en lengua toscana en múltiples bibliotecas de los siglos XVI y XVII. Es más, creemos que la vía de permeabilización y difusión del incipiente maquiavelismo fue mucho más amplia que la demostrada por medio de las referencias bibliotecarias.

Por otro lado, tanto la actitud de la Iglesia romana como la de la Inquisición española no fueron inicial ni unívocamente opuestas a los planteamientos maquiavelianos, ni en el mismo momento ni con la misma contundencia, y este hecho facilitó la transmisión de la obra y las concepciones políticas de nuestro autor. Así lo demuestra conceptualmente la temática desarrollada por ciertos hombres de letras españoles ${ }^{26}$ de gran relevancia en los siglos XVI y XVII en torno, por ejemplo, al realismo político como contrapunto al utopismo naciente; la experiencia política personal; las relaciones entre política y ética; o la razón de Estado; la educación del príncipe; la historia como magistra vitae; la obtención y observancia de la virtud; el arbitrario devenir de la fortuna; la adulación y la falsedad; la simulación y la disimulación; el estudio de los tipos de gobierno más deseables; la relación entre fines y medios; la cuestión acerca de la naturaleza humana y la constitución del Estado; el papel de la religión en la política; la legitimidad, etc. Y aun cuando las redes inquisitoriales cayeran sobre la obra de Maquiavelo, ni fue esta la mayor de sus preocupaciones ni pudieron atraparla absolutamente, pues es evidente que permeabilizó. En consecuencia, los argumentos y criterios expuestos que niegan la presencia, el impacto y la influencia de la obra y el pensamiento de Maquiavelo en España durante los siglos XVI y XVII resultan insuficientes. Afirmamos la pervivencia y el impacto

26 Véase la nota 1. 
del pensamiento maquiaveliano, así como las categorías y conceptos que se derivan de su obra, tal como recogen diversos autores de relevancia y prestigio para la literatura política española en los siglos XVI y XVII.

ReCIBIDO: septiembre 2019; ACEPTADO: diciembre 2019 